\title{
Supporting Information \\ Fuel Sulfur and Iron Additives Contribute to the Formation of Carbon Nanotube-like Structures in an Internal Combustion Engine
}

\author{
Jacob J. Swanson ${ }^{1,2,}$, Ryder Febo ${ }^{1,2}$, Adam Boies ${ }^{3,4}$, David B. Kittelson ${ }^{2}$ \\ ${ }^{I}$ Department of Integrated Engineering, Minnesota State University Mankato, Mankato, MN 55423, USA \\ ${ }^{2}$ Department of Mechanical Engineering, University of Minnesota, Minneapolis, MN 55455, USA \\ ${ }^{3}$ Department of Civil, Environmental, and Geo- Engineering, University of Minnesota, Minneapolis, MN 55455, USA \\ ${ }^{4}$ Department of Engineering, University of Cambridge, Cambridge, CB2 1PZ, United Kingdom \\ *E-mail address: jacob.swanson@mnsu.edu
}

\section{Experimental apparatus}

Figure S1 shows the experimental apparatus.

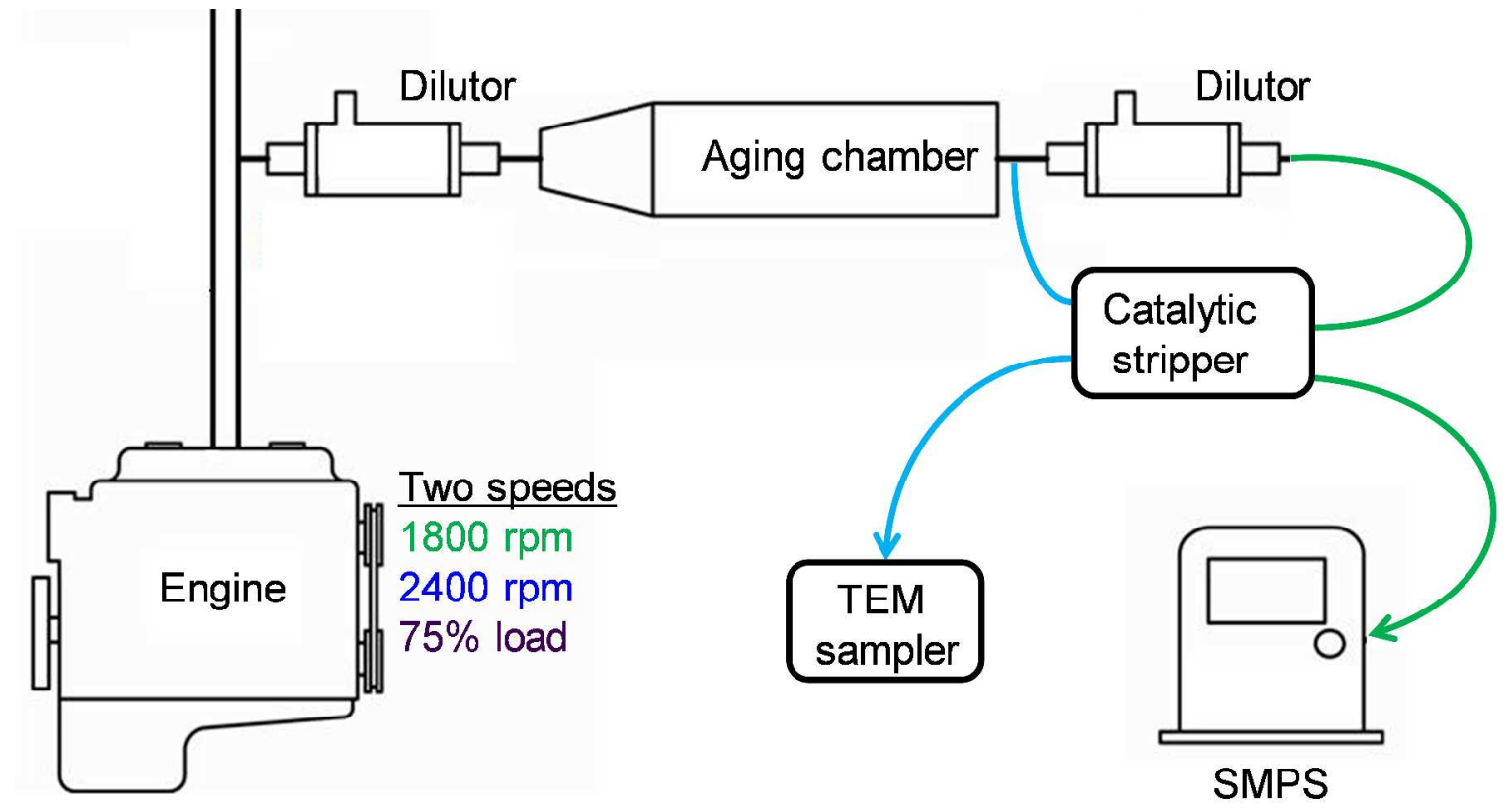

Figure S1. Experimental apparatus for diesel particle generation and sampling. SMPS measurements were conducted both with and without the catalytic stripper.

\section{CNT-like structures identified for Conditions 1-4}

For all but Condition 5 (4500 ppm S, 36 ppm Fe) CNT-like structures were sparsely distributed, poorly defined, and small. Figure S2 shows three examples of particles that were counted for the zero Fe, zero S condition. They are much less "CNT-like" and shorter than the structures seen in 
Condition 5, but there is a hint of CNT-like growth, and they do meet the conditions we imposed (i.e. diameter $<15 \mathrm{~nm}$, aspect ratio $>3: 1$ ). A few CNTs might be expected, however, since there was a small amount of sulfur in the fuel (10 ppm) and oil consumption of the engine is higher than a modern diesel engine. High oil consumption naturally leads to the formation of metal nanoparticles, some of which may be iron resulting from additives of wear.

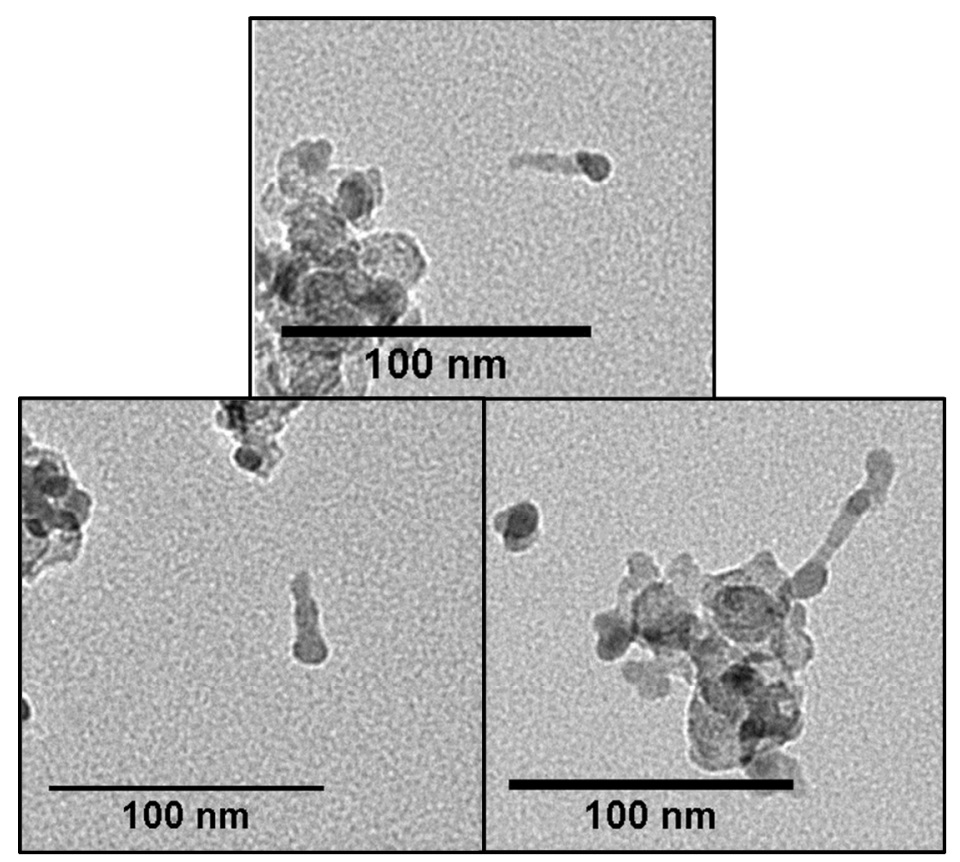

Figure S2. Less-defined CNT-like structures for Condition 1 (zero S, zero Fe addition).

\section{"Decorated" carbonaceous aggregates}

It is known that the combustion of metal additives in lubrication oil or fuel leads to formation of solid metal oxide nanoparticles. These are found in the nucleation mode when oil consumption is high and soot concentrations are low, (typically at idle or low load conditions) or decorated on carbonaceous aggregates. ${ }^{1-4}$ Figure $\mathrm{S} 3$ shows decorated carbonaceous aggregates from the zero $\mathrm{Fe}$, zero $\mathrm{S}$ condition. Ash particles are nearly spherical and $\sim 5 \mathrm{~nm}$ to $10 \mathrm{~nm}$ in diameter. Typical ash particle metals are calcium, zinc, phosphate, magnesium, and manganese. ${ }^{5}$ The presence of these particles demonstrates that oil consumption for this engine, even at high load, was high enough to promote significant metal nanoparticle nucleation. Such particles would be present for CNT formation, which may partially explain the observance of spurious CNT-like structures in Conditions $1-4$. 


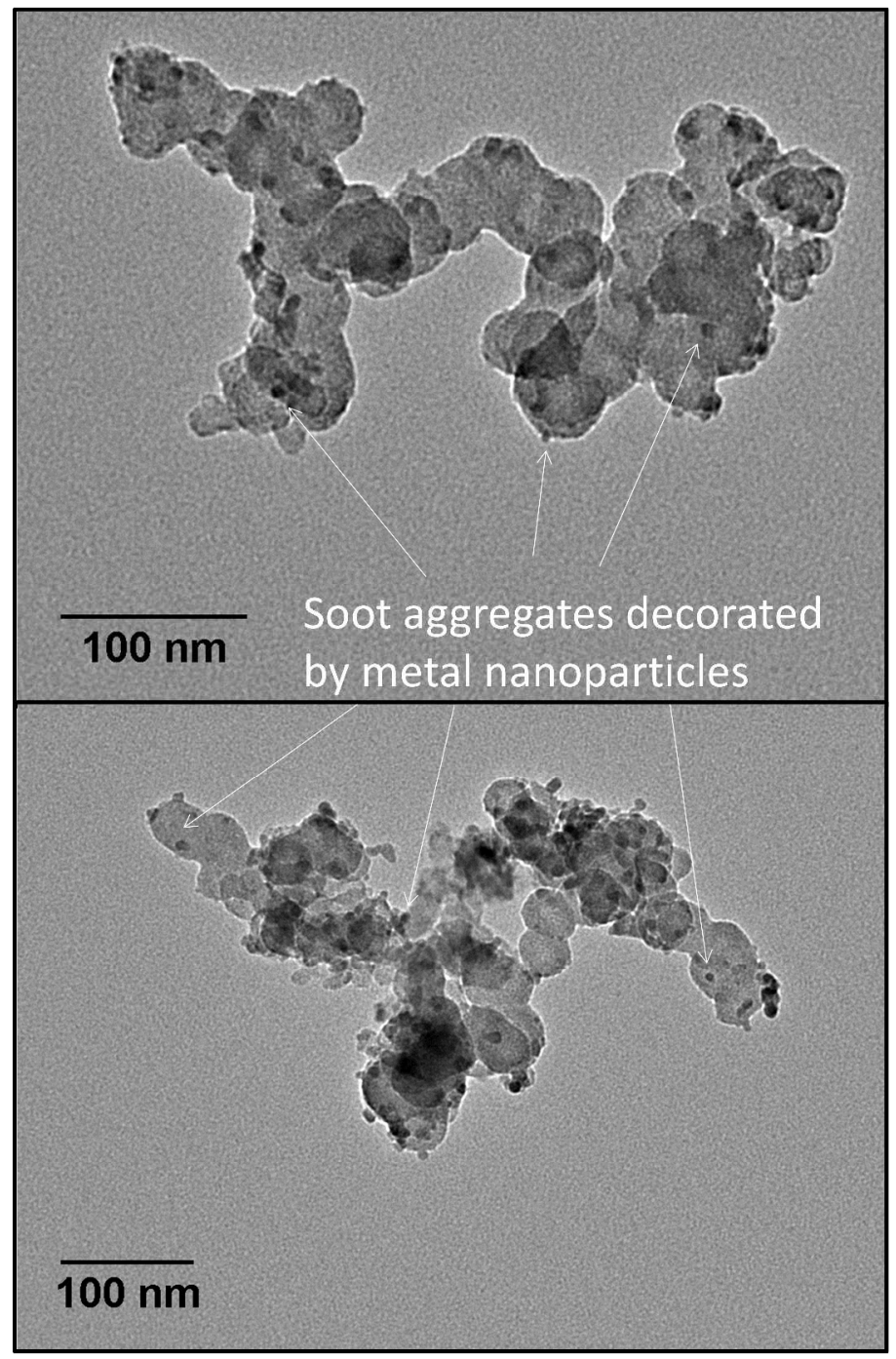

Figure S3. Carbonaceous aggregates particles decorated by metal nanoparticles.

\section{Particle size distributions}

Exhaust aerosol size distributions were measured with a TSI model 3080 scanning mobility particle sizer (SMPS) consisting of a TSI model 3081 differential mobility analyzer (DMA,) and a TSI model 3776 ultrafine condensation particle counter (CPC). The SMPS was operated over a size range of $6 \mathrm{~nm}$ to $225 \mathrm{~nm}$ with the sheath and aerosol flows set to 15 and $1.5 \mathrm{~L} / \mathrm{min}$, respectively. All measurements included a minimum of three SMPS scans. The SMPS was used with and without a catalytic stripper that was used to remove the organic carbon fraction by passing the dilute diesel exhaust through a catalytic stripper ("CS", model CS015, Catalytic Instruments $\mathrm{GmbH}$ ) heated to $350^{\circ} \mathrm{C}$. The $\mathrm{CS}$ was placed in the sampling train upstream of the 
TEM sampler and SMPS but downstream of the dilution system. All data is corrected for sizedependent CS losses.

Results shown in Figures S4 and S5 illustrate the following.

1) For all conditions, there is a tremendous amount of semi-volatile material present, evidenced by a factor of $10 \times$ or more reduction in particle number concentration with the use of the CS.

2) There are solid nucleation mode particles present at all conditions (even without Fe addition) that could potentially serve at CNT nucleation sites. Given the high oil consumption of this particular engine, the amount of other metals in the system was so high, a distinct nucleation mode related to the addition of Fe was not apparent.

3) The addition of Fe significantly (one order of magnitude or more) reduces the soot number concentration (and therefore mass) in the accumulation mode. Fe is probably acting late in the combustion process, after it has decomposed, nucleated to form nanoparticles, and diffused to the surface of soot, to catalytically react with soot particles to reduce their size and concentration. Since ferrocene is a common fuel-borne catalyst, this behavior is expected.

4) Consistently and for all conditions, the addition of $4500 \mathrm{ppm} \mathrm{S}$ significantly reduces the soot number (and therefore mass) in the accumulation mode. The response to $500 \mathrm{ppm} \mathrm{S}$ was mixed. Due to the known interaction of S and carbon in gas-phase CNT processes, and the fact that reacting carbon and $\mathrm{S}$ is a standard manufacturing process for the production of carbon disulfide $\left(\mathrm{CS}_{2}\right)$, it is suspected that the reduction of soot with high levels of $\mathrm{S}$ is real and perhaps warrants further investigation. 

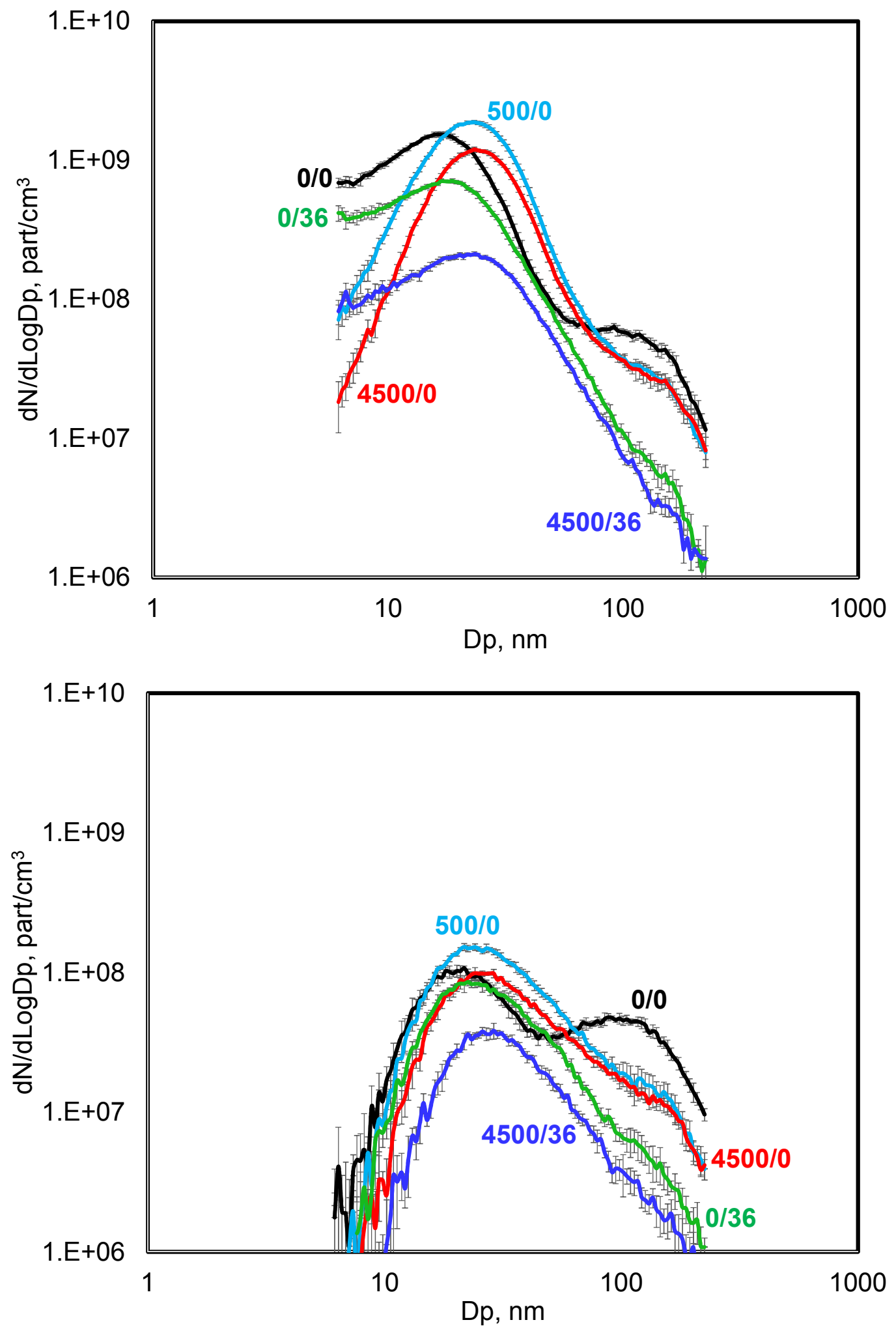

Figure S3. Particle size distributions for the $1800 \mathrm{rpm}$, high load condition. Top = with catalytic stripper, bottom = without catalytic stripper (solid particles only). Error bars represent the standard deviation of five SMPS scans. Conditions are identified by ppm S / ppm Fe. 

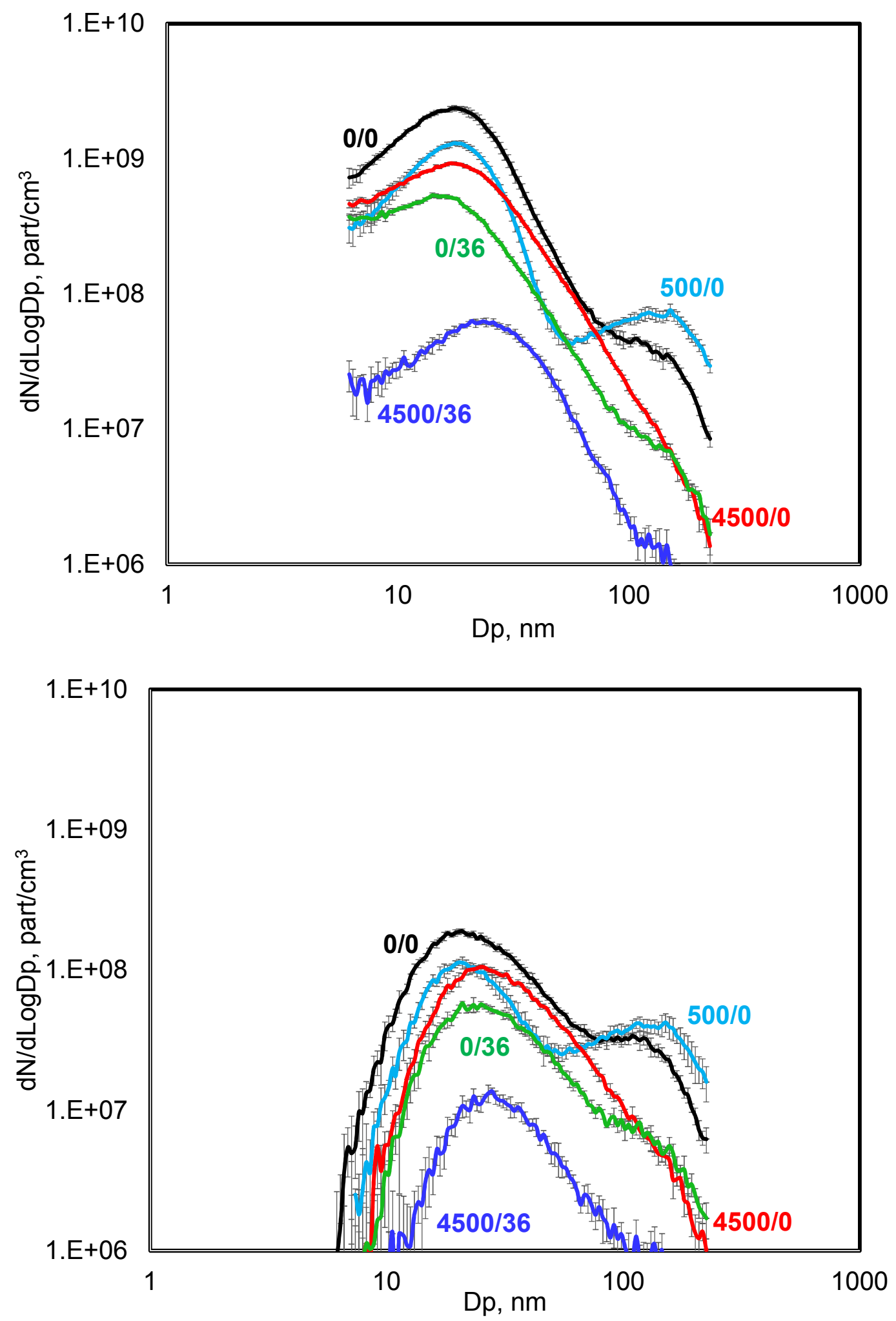

Figure S5. Particle size distributions for the $2400 \mathrm{rpm}$, high load condition. Top = with catalytic stripper, bottom $=$ without catalytic stripper (solid particles only). Error bars represent the standard deviation of five SMPS scans. Conditions are identified by ppm S / ppm Fe. 


\section{Additional particle and CNT-like structure statistics}

Figures S6 and S7 show total particle number and total CNT-like structure number for all conditions. It is notable that fewer particles were counted for the high $\mathrm{S}$ high Fe condition. This is because the sampling conditions (e.g. voltage, sampling time and flowrate) were kept constant, and as shown by the particle size distributions, this condition contains the lowest particle concentration. The ratio of the number of CNT-like structures to total number of particles should not be taken to represent the fraction of CNT-like structures emitted by the engine since some particles clearly aggregated on the grid and were only counted as one.

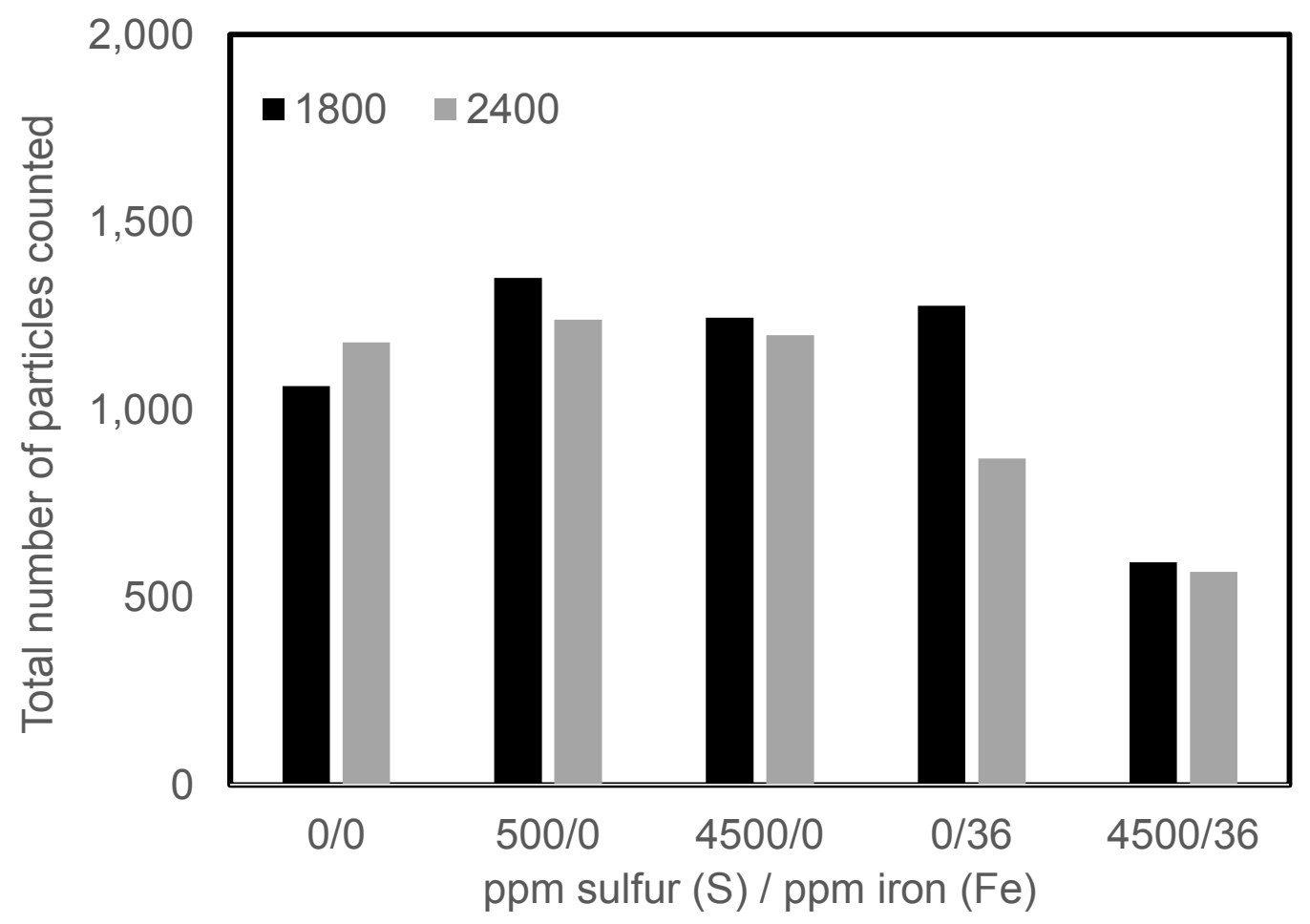




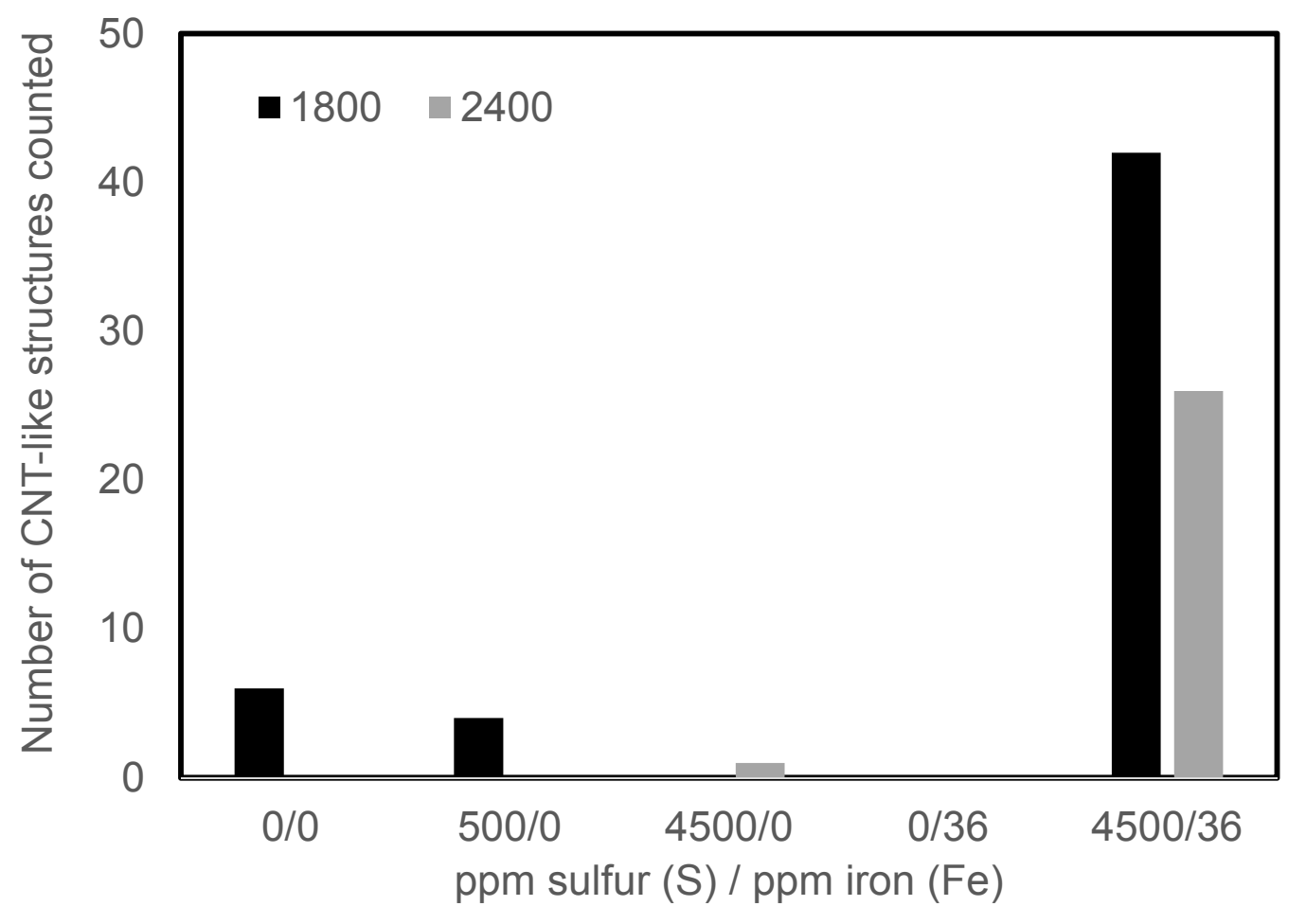

\section{REFERENCES}

1. Kittelson, D. B.; Watts, W. F.; Johnson, J. P. Diesel aerosol sampling methodology. CRC E-43 Final Report. http://www.me.umn.edu/centers/mel/reports/crce43ts.pdf. 2002, Accessed 22 May, 2016.

2. Kittelson, D. B.; Watts, W. F.; Johnson, J. P. On-road and laboratory evaluation of combustion aerosols - Part 1: summary of diesel engine results. J. Aerosol Sci. 2006, 37, 013930.

3. Jung, H.; Kittelson, D. B.; Zachariah, M. R. The influence of a cerium additive on ultrafine diesel particle emissions and kinetics of oxidation. Combustion Flame 2005, 142, 276-288.

4. Mayer, A.; Ulrich, A.; Czerwinski, J.; Mooney, J., Metal-oxide particles in combustion engine exhaust. SAE Technical Paper 2010, 2010-01-0792.

5. Barris, M. A.; S. B. Reinhart, S.B.; Wahlquist, F. H. The Influence of lubricating oil and diesel fuel on ash accumulation in an Exhaust Particulate Trap, SAE Technical Paper Series, 1991, 910131. 